\title{
Probing heavy ion collisions using quark and gluon jet substructure
}

\author{
Yang-Ting Chien*t \\ Center for Theoretical Physics, Massachusetts Institute of Technology, Cambridge, MA 02139, \\ U.S.A. \\ E-mail: ytchien@mit.edu
}

\begin{abstract}
Understanding the inner working of the quark-gluon plasma requires complete and precise jet substructure studies in heavy ion collisions. In this proceeding we discuss the use of quark and gluon jets as independent probes, and how their classification allows us to uncover regions of QCD phase space sensitive to medium dynamics. We introduce the telescoping deconstruction (TD) framework to capture complete jet information and show that TD observables reveal fundamental properties of quark and gluon jets and their modifications in the medium. We draw connections to soft-drop subjet distributions which help illuminate medium-induced jet modifications. The classification is also studied using a physics-motivated, multivariate analysis of jet substructure observables. Moreover, we apply image-recognition techniques by training a deep convolutional neural network on jet images to benchmark classification performances. We find that the quarkgluon discrimination performance worsens in JEWEL-simulated heavy ion collisions due to significant soft radiation affecting soft jet substructures. This work suggests a systematic framework for jet studies and facilitates direct comparisons between theoretical calculations and measurements in heavy ion collisions.
\end{abstract}

International Conference on Hard and Electromagnetic Probes of High-Energy Nuclear Collisions 30 September - 5 October 2018

Aix-Les-Bains, Savoie, France

\footnotetext{
* Speaker.

${ }^{\dagger}$ The author would like to thank the Hard Probes 2018 organizers for the hospitality and support.
} 

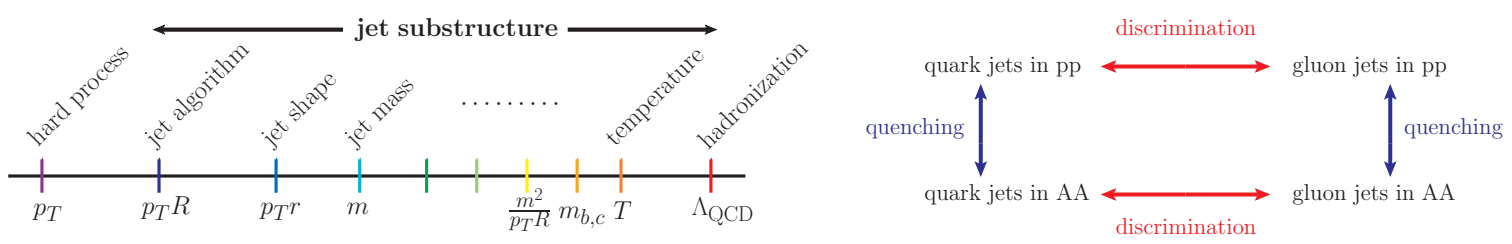

Figure 1: Left panel: Jet substructure observables can probe QCD dynamics in all energy regimes from the highest scale down to $\Lambda_{\mathrm{QCD}}$ [1]. Right panel: Classification of quark and gluon jets in pp and AA collisions provide a new method for jet quenching studies [3].

\section{Introduction}

The study of jet quenching has moved onto detailed analysis of the redistribution of jet energy quantified by jet substructure modifications. Different jet substructure observables are sensitive to different underlying QCD dynamics at characteristic energy scales (left panel of Fig. 1). One can design jet substructure observables to probe specific regions of phase space where jet-medium interaction may have the dominant effect. A comprehensive examination of jet substructure modifications will then allow us to search for possible signatures which may reveal fundamental properties of the quark-gluon plasma (QGP). On the other hand, a change of quark and gluon jet fractions in heavy ion collision can contribute significantly to jet substructure modifications. An increase of the quark-jet fraction due to larger suppressions of gluon jets can make the jet energy profile more quark-jet like, an important effect in addition to the jet-by-jet modification to substructure $[1,2]$. This further motivates the studies of jet modifications with different quark and gluon jet fractions which enable the use of quark and gluon jets as independent probes.

In this proceeding we exploit this idea and study classifications of quark and gluon jets in $p p$ and $A A$ collisions. The goal is to extract complete jet features which encode all aspects of jet modifications in $A A$ collisions. We study the discrimination of jets in $p p$ and $A A$ collisions and show that it is intimately related to quark-gluon discrimination (right panel of Fig. 1) which aims to identify differences between quark and gluon jets. We use three approaches, starting from a multivariate analysis of a list of physics-motivated jet observables (left panel of Fig. 2). On the other hand, we apply image recognition techniques which identify relevant features using machine learning methods (middle panel of Fig. 2). In between, we introduce the telescoping deconstruction framework which aims to organize and capture complete physical information within jets using telescoping subjets (right panel of Fig. 2). Below we briefly summarize each of the method.

\section{Quark and gluon jet substructure and modification}

The quark and gluon enriched jet samples used in this work were generated using the prompt photon production channels $q+\gamma$ and $g+\gamma$ in JEWEL. The physics-motivated, multivariate analysis combines information captured in each individual jet observable. We consider five representative ones: jet mass, radial moments, $p_{T}^{D}$ and pixel multiplicity. We see that gluon jets have broader energy distributions and softer hadron fragmentation compared to quark jets, and medium interactions result in broader energy distribution and softer hadron fragmentation for both quark and gluon 

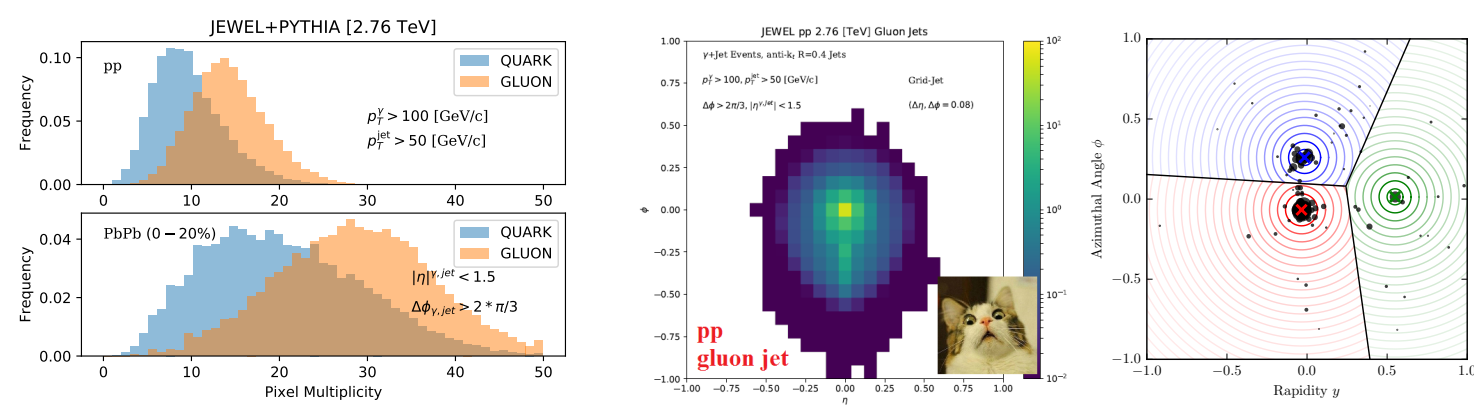

Figure 2: Left panel: Pixel multiplicity distributions for quark and gluon jets in $p p$ (upper) and $A A$ (lower) collisions simulated using JEWEL. Middle panel: Average gluon jet image in $p p$ collisions. Right panel: Telescoping deconstruction of a QCD jet at the T3 order.

jets. The jet image method trains a deep convolutional neural network $(\mathrm{CNN})$ on quark and gluon jet images in $p p$ and $A A$ collisions [4]. The energy distribution in rapidity $y$ and azimuthal angle $\phi$ is discretized with a finite pixel size. The $\mathrm{CNN}$ is then capable of processing raw pixel jet data and finding useful features which help maximize the separation among jet samples. From the average jet images, we see again that gluon jets are more spread out and populating more pixels with soft particles compared to quark jets, and the medium further broadens the energy distribution.

The TD framework probes energy flows within jets using subjets with multiple angular resolutions [5, 6]. It decomposes jet information in a fixed-order expansion organized by the number of reconstructed subjets. At the TN order, the procedure starts from identifying $N$ dominant energy flow directions along soft-recoil-free axes. Subjets are then reconstructed around the axes with multiple subjet radii $R_{T}$, and subjet kinematic variables form a complete jet substructure basis. We show that subjet momentum fraction $z$ and angular distributions $\theta$ constructed in telescoping deconstruction encode fundamental QCD properties such as the Altarelli-Parisi splitting functions (Fig. 3), similar to the groomed momentum sharing $z_{g}$ and groomed jet radius $r_{g}$ constructed in Soft Drop [7]. Note the characteristic $1 / z$ functional form in the subjet momentum fraction. Recently the soft-drop $z_{g}$ variable was used to probe heavy ion collisions with significant enhancement of soft subjets [8] which was first explained as a signature of medium-induced radiation [9]. We see a similar modification pattern in the $z$ distribution and also show that the $\theta$ distribution receives strong medium modifications enhancing wide-angle emissions. To go beyond, we examine subjet masses which reveal the flavor origin of quark and gluon jets, with significant modification in $A A$ collisions. This is further tested using a collinear-drop observable $\delta m$ which is designed to probe soft radiation within jets (lower panels of Fig. 3). We see that the difference between quark and gluon jets in $\delta m$ disappears in $A A$ collisions, which suggests that soft radiation washes out such feature that distinguishes quark and gluon jets, a possible signature of medium response to jets.

\section{Quark and gluon jet classification}

Having examined the jet substructure information represented using physics-motivated observables, jet images and TD basis, we combine all the information in each category using multivariate analysis tools and study the classification of quark and gluon jets in $p p$ and $A A$ collisions. A 

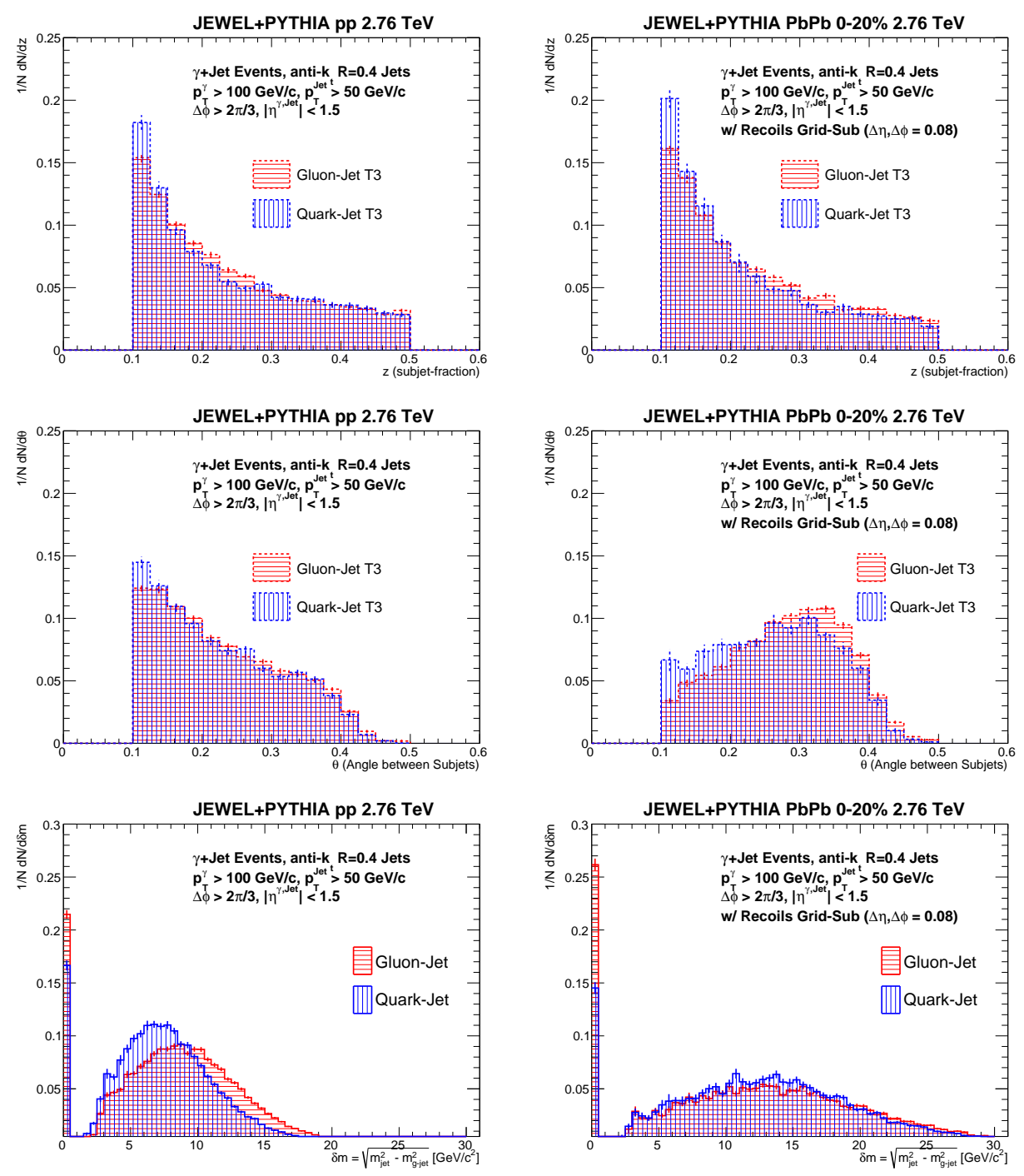

Figure 3: Upper panels: Subjet momentum fraction distributions at the T3 order for quark and gluon jets in $p p$ (left) and $A A$ (right) collisions. Middle panels: Subjet angular distributions at the T3 order for quark and gluon jets in $p p$ (left) and $A A$ (right) collisions. Lower panels: Distributions of the collinear-drop observable $\delta m$ for quark and gluon jets in $p p$ (left) and $A A$ (right) collisions

proper neural network architecture is chosen for processing the simulated input data. We perform two tasks, discriminating quark jets and gluon jets, and discriminating jets in $p p$ and $A A$ collisions. We quantify the classification performance using receiver-operating-characteristic (ROC) curves, plotting signal efficiency versus background efficiency (Fig. 4) with higher performance towards the lower-right corner of the plots. We see that all methods give consistent and comparable performance, suggesting that with the discretization resolution each method captures most of the substructure information. The telescoping deconstruction performance converges quickly with increasing $\mathrm{T} N$ order. We find that the quark-gluon discrimination performance goes down in JEWEL-simulated $A A$ collisions. Also, the pixel multiplicity is the dominant observable distinguishing jets in $p p$ and $A A$ collisions, a characteristic feature of the significant soft event activities. 

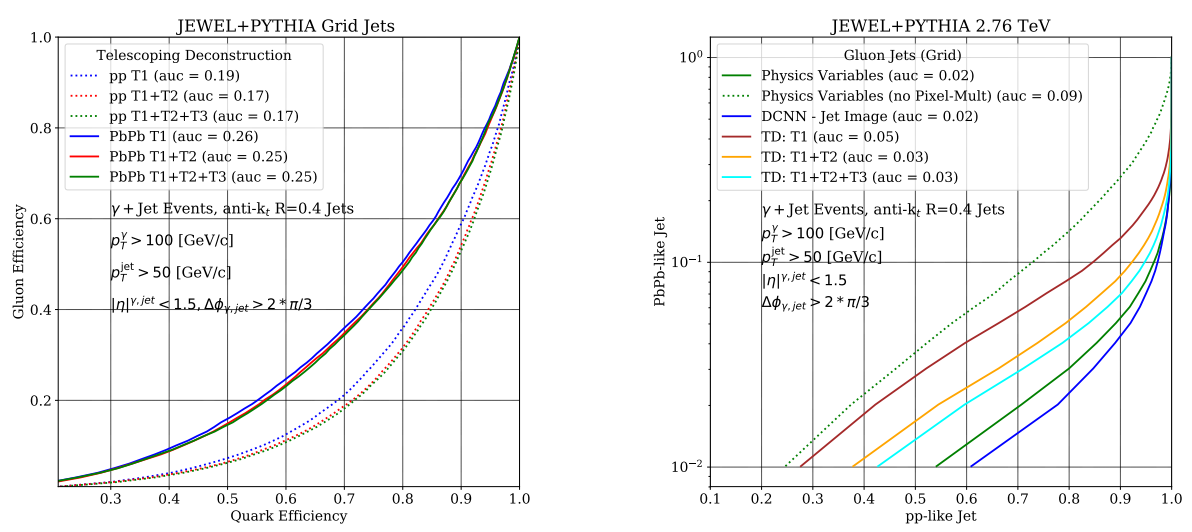

Figure 4: Left panel: ROC curves using TD variables for quark-gluon discrimination in $p p$ and $A A$ collisions. Right panel: ROC curves using physics-motivated multivariate analysis, jet image and TD for discriminating gluon jets in $p p$ and $A A$ collisions.

\section{Conclusions}

We show that quark and gluon jet substructure can be independent probes of jet-medium interaction and that quark-gluon discrimination is a new way for jet modification studies. We use physics-motivated multivariate analysis and machine learning tools, and we develop the TD framework to decompose jet information using subjet basis. We emphasize that comprehensive substructure studies can lead to the understanding of the inner working of QGP.

\section{References}

[1] Y. T. Chien and I. Vitev, Towards the understanding of jet shapes and cross sections in heavy ion collisions using soft-collinear effective theory, JHEP 1605, 023 (2016)

[2] S. Chatrchyan et al. [CMS Collaboration], Modification of jet shapes in $\mathrm{PbPb}$ collisions at $\sqrt{s_{N N}}=2.76 \mathrm{TeV}$, Phys. Lett. B 730, 243 (2014)

[3] Y. T. Chien and R. Kunnawalkam Elayavalli, Probing heavy ion collisions using quark and gluon jet substructure, arXiv:1803.03589 [hep-ph].

[4] P. T. Komiske, E. M. Metodiev and M. D. Schwartz, Deep learning in color: towards automated quark/gluon jet discrimination, JHEP 1701, 110 (2017)

[5] Y. T. Chien, Telescoping jets: Probing hadronic event structure with multiple R's, Phys. Rev. D 90, no. 5, 054008 (2014)

[6] Y. T. Chien, A. Emerman, S. C. Hsu, S. Meehan and Z. Montague, Telescoping jet substructure, arXiv:1711.11041 [hep-ph].

[7] A. J. Larkoski, S. Marzani, G. Soyez and J. Thaler, Soft Drop, JHEP 1405, 146 (2014)

[8] A. M. Sirunyan et al. [CMS Collaboration], Measurement of the Splitting Function in $\mathrm{pp}$ and $\mathrm{Pb}-\mathrm{Pb}$ Collisions at $\sqrt{s_{\mathrm{NN}}}=5.02 \mathrm{TeV}$, Phys. Rev. Lett. 120, no. 14, 142302 (2018)

[9] Y. T. Chien and I. Vitev, Probing the Hardest Branching within Jets in Heavy-Ion Collisions, Phys. Rev. Lett. 119, no. 11, 112301 (2017) 